\title{
Matching Pursuit for Sparse Signal Reconstruction Based on Dual Thresholds
}

\author{
Zhengguang Xie*, Huang Hongwei, Cai Xu \\ School of Electronics and Information, Nantong University, Nantong City, Jiangsu Province, China 226019. \\ * Corresponding author. Tel.: 86-18206294387; email: xie_zg@126.com \\ Manuscript submitted January 10, 2015; accepted December 15, 2015. \\ doi: 10.17706/ijcce.2016.5.5.341-349
}

\begin{abstract}
Anumberofsparserecoveryapproacheshaveappearedintheliterature based on Orthogonal Matching Pursuit (OMP) algorithms because of its low computationalComplexity. Thismanuscriptintroducesanoveladaptive forward-back greedy approach, called Dual Threshold Matching Pursuit (DTMP), which select atoms based on two appropriate thresholds. During forward atom increasing process, DTMP picks out new candidate atoms based on the forward threshold under Restricted Isometry Constant (RIC) condition. In backward atom decreasing process, DTMP deletes wrong atoms based on the backward threshold according tothe principal of energy concentration. Like forward-backward pursuit (FBP), DTMP does not need the sparsity level in contrast to the Subspace Pursuit (SP) or Compressive Sampling Matching pursuit (CoSa MP) algorithms. Experimental results show that the reconstruction accuracy of DTMP surpasses SP, FBP and other greedy algorithms obviously and its complexity is comparable with those of OMP and SP.
\end{abstract}

Key words: Compressed sensing, greedy algorithm, support update, dual thresholds.

\section{Introduction}

The main content of compressed sensing (CS) [1]-[4] theory is that the main information of the original signal is contained in a small amount of linear projection of compressible signal and original signal can be reconstructed accurately by global linear measuring. In the CS theoretical frame, consider 1D (one-dimensional) signal $\mathbf{x} \in R^{N}$ of length of $N$ that has only $K$ nonzero entries with $K \ll N$. That is, the process of signal measurement can be expressed by Equation $\mathbf{y}=\Phi \mathbf{x}$, where $\mathbf{y}$ represents the observation signal of length $M, \Phi=\left[\varphi_{1}, \varphi_{2}, \ldots, \varphi_{N}\right]$ represents the observation matrix with size of $M \times N$ and $\mathbf{x}$ denotes the $k$-sparse signal $(K<M<N)$. This process is equivalent to the process that the original signal $\mathbf{x}$ is projected onto the space spanned by the $\left[\varphi_{1}, \varphi_{2}, \ldots, \varphi_{N}\right]$. Consequently, the size of signal decreases from $N$ dimension to $M$ dimension. From the size of $\Phi$ and $\mathbf{y}$ in equation (1), we can see that the equation is underdetermined. So the problem to solve the equation is ill-posed. However, we can reconstruct the signal precisely when the signal contains limited nonzero entries. Candes and Donoho [1]-[4] pointed out that it is not necessary to resort to $l_{0}$ optimization to recover $\mathbf{x}$ from the underdetermined inverse problems; many reasonable $l_{1}$ optimization algorithms [5] yield an equivalent solution only if $\Phi$ satisfies Restricted Isometry Property (RIP) [6] with a constant parameter which is defined as follows: 


$$
\left(1-\delta_{K}\right)\|x\|_{2}^{2} \leq\|\Phi x\|_{2}^{2} \leq\left(1+\delta_{K}\right)\|x\|_{2}^{2}
$$

where $\|\bullet\|_{2}$ stands for the $l_{2}$ vector norm.

However, the computational complexity of linear programming techniques is too high to put CSinto practical application [4]. Therefore, the initial greedy algorithm, such as OMP [7], has attracted quiet more attention with its simple structureand low computational complexity, since it only add atoms to its support per iteration. In other words, OMP has only forward step at each iteration. Due to low reconstruction accuracy by OMP, some improved algorithms based on OMP, such as SP [8], CoSaMP [9] and FBP [10], can achieve high reconstruction accuracy by adding the strategy of backtracking.

According to the step lengthof forward and backward, these forward-back greedy algorithms can be classified into two categories, the non-adaptive algorithms and adaptive algorithms. For non-adaptive algorithms, the forward and backward stepsize areconstant $\mathrm{K}$ for SP, constant $2 \mathrm{~K}$ for CoSaMP, as well as constant $\alpha$ and $\beta$ for FBP. The adaptive algorithmswhose step size are variable include adaptive sparse matching pursuit (ASMP) [11], adaptive threshold backtracking orthogonal matching pursuit (ATBOMP) [12], and others similar algorithms [13], [14].

Generally speaking, the accuracy of variable step algorithms should be higher than those of constant step algorithms, if the step length of the former depended on reasonable conditions [15]. However, lots of experimental results show that the performance of FBP is better than those of ASMP and ATBOMP which will be presented in the second part of this paper. The target of this paper is to investigate the differences among these algorithms and furtherly to propose a noveladaptive forward-back greedy algorithm to improve the performance of variable step algorithms.

\section{Description of FBP, ASMP and ATBOMP}

Before proceeding, we set our notations through whole our paper. $\operatorname{supp}(\mathbf{x})$ denotes the indexes of nonzero elements in vector $\mathbf{x} . K$ represents sparsity level of the target signal. $\Phi^{*}$ and $\Omega$ are the transposition and whole set of $\Phi$, respectively. $\Lambda^{0}$ stands for initial support and $\mathbf{r}^{0}$ denotes the initial signal residual. $\Lambda^{l}$ and $\mathbf{r}^{l}$ denote the signal support and signal residual respectively after $l$-th iteration. $\left|\Lambda^{l}\right|$ is the number of elements in $\Lambda^{l}$. By $\mathrm{x}_{\Lambda^{l}}$ we mean the set of $\mathrm{x}$ indexed by $\Lambda^{l} . \delta_{K+1}$ denotes the RIP constant of order $K+1 .<\bullet>$ stands for inner product operator. $\mathbf{h}^{l}(i)=<\varphi_{i}, r^{l-1}>$ is equal to $i$-th elements of proxy signal for residual $\mathbf{r}^{l-1} \cdot e_{i}$ stands for $i$-th column of unit matrix. Average $(\bullet)$ is average operator.

Unlike original greedy algorithms, FBP, ASMP and ATBOMP can take advantage of backtracking to refine the chosen supports. FBP contains two core processes in every iteration. During forward process, the $\alpha$ indexes (named forward step size) which are maximally correlated with the residual are picked up to expand the support. The observation signal $\mathrm{y}$ is projected onto the subspace spanned by the support obtained during forward process. Atoms corresponding to $\beta$ (called the backward size) minimum contribution to this projection are removed to produce the final support estimate $\Lambda^{l}$ of the $l$-th iteration. Afterwards, the observation signal is projected onto the final support in this iteration and the residual is re-calculated. These two core processes will be continued until the energy of residual is less than a very small value $\varepsilon$ or $\left|\Lambda^{l}\right|$ is greater than constant $l_{\max }$.

ASMPalgorithm consists of two nested iterations: the outer one and the inner one. The objective of the outer loop is to estimate the sparsity based on a constant and residual. With the estimated sparsity level, the inner loop reconstruct the target signal using backtracking principle. That is to say, ASMP selects atoms 
with a threshold calculated by the energy of residual and a constant, and then construct its support according to its sparsity estimated in current iteration. This nested process is repeated until the residual energy is smaller than a certain threshold $\varepsilon$.

ATBOMP includes support selection and support final decision per iteration. Support set is chosen by adaptive threshold and regularized selection. The candidate set is decided by the threshold $\mu$ which is adaptively set according to the residual and the measurement matrix $\Phi$. In final selection, a regularized procession is used to remove some atoms chosen wrongly in the previous processing. Its iteration will stop when the current residual's 12 norm is smaller than a threshold $\varepsilon$ or when the maximum number of iterations $l_{\max }$ is reached.
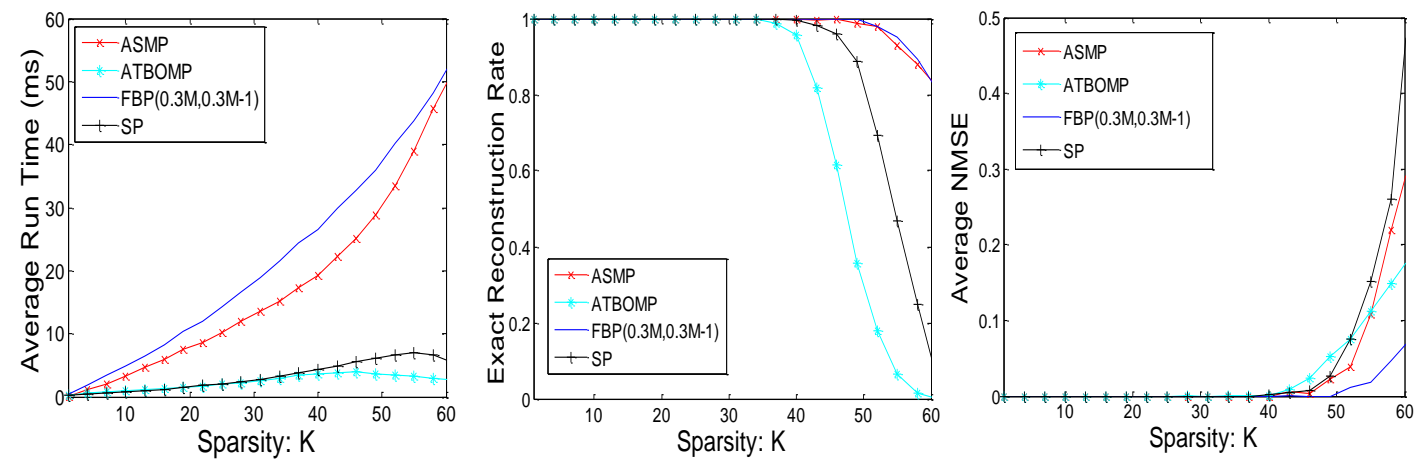

Fig. 1. Reconstruction results with existed algorithms for the Gaussian sparse signal.
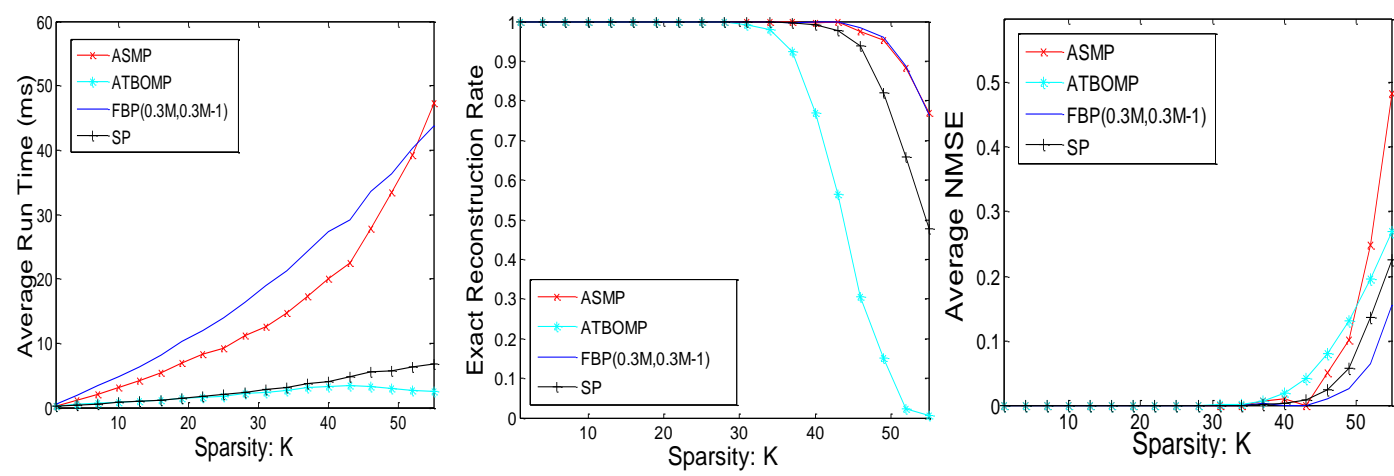

Fig. 2. Reconstruction results with existed algorithms for the uniform sparse signal.

The time cost of Sparsity Adaptive Matching Pursuit (SAMP) [13] algorithm becomes large when K is big, while the step size of SAMP is fixed which may cause overestimation or underestimation. The thresholds in the Backtracking-based Matching Pursuit (BAOMP) [14] need to be preset artificially. If choosing inappropriate thresholds, the performance will degrade greatly. Zhao [12] has point out the performance of ASMP is better than that of SAMP and BAOMP. Therefore, the following experiment is only compared the performance of SP, FBP, ATBOMP and ASMP algorithms.

In general, the accuracy of adaptive algorithms should be higher than that of non-adaptive ones, if the step length of the former are appropriate. However, Fig. 1-Fig. 3 shows that the performance of FBP is better than that of ASMP and ATBOMP (The experimental environment is the same as that of Section 4).

Fig. 1-Fig. 3 shows the Gaussian, uniform and 0-1 sparse signals reconstruction results obtained by ASMP, ATBOMP, FBP and SP algorithms. In the second picture of each figure, we present the exact recovery rate, which is the rate of the number of exactly reconstruction to the number of total test. The exactly 
reconstruction condition is $\|\mathbf{x}-\hat{\mathbf{x}}\|_{2} \leq 10^{-2}\|\mathbf{x}\|_{2}$. In the third picture, the recover error is calculated by Average Normalized Mean-squared-error, which is defined as ANMSE $=\frac{1}{n} \sum_{i=1}^{n}\left\|\mathbf{x}_{i}-\mathbf{x}_{i}\right\|_{2}^{2} /\left\|\mathbf{x}_{i}\right\|_{2}^{2}$, where $n$ is the number of total test. FBP $(0.3 \mathrm{M}, 0.3 \mathrm{M}-1)$ represents FBP with forward step length $\alpha=0.3 \mathrm{M}$ and back step length $\beta=\alpha-1$, which should be the best step lengths according to [11].
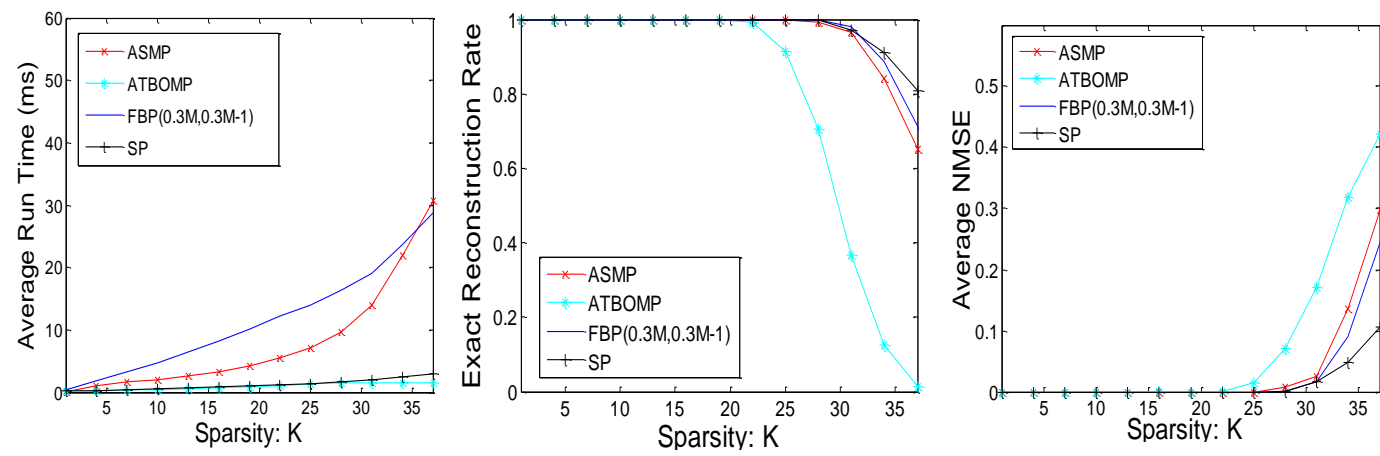

Fig. 3. Reconstruction results with existed algorithms for the ' $0-1$ ' sparse signal.
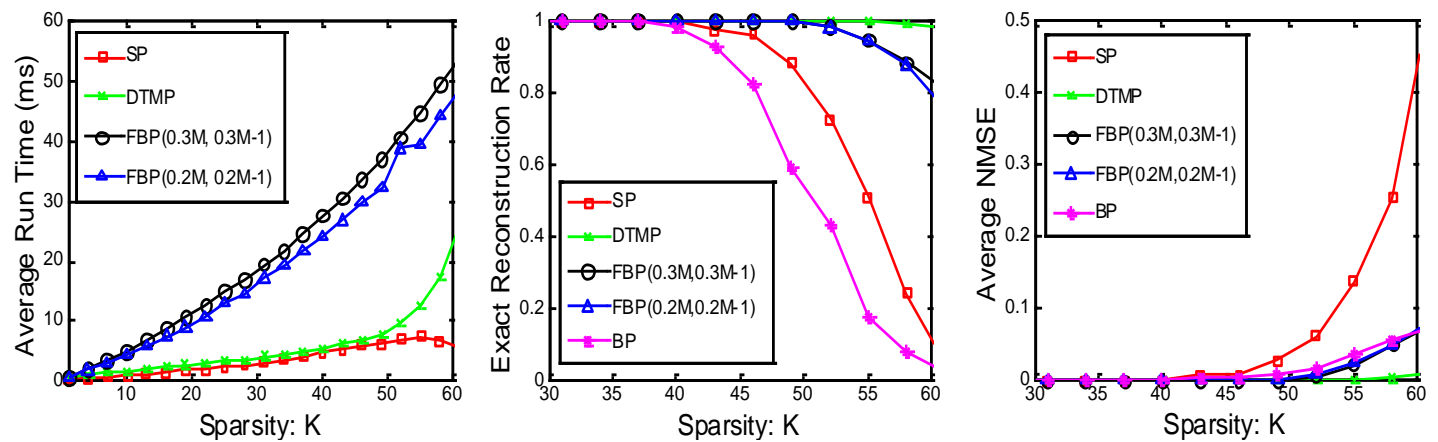

Fig. 4. Reconstruction results over sparsity for the Guassian sparse signal.

From Fig. 1, we observe that the exact recovery rate of FBP $(0.3 \mathrm{M}, 0.3 \mathrm{M}-1)$ is the best among all the four algorithms, which starts to fail at around $K=50$. ASMP fails a little earlier, around $K=45$. Both of FBP $(0.3 \mathrm{M}$, 0.3M-1) and ASMP are significantly better than ATBOMP and SP at exact reconstruction rate at the cost of higher reconstruction time. As for ANMSE, FBP (0.3M, 0.3M-1) is the best performer among the four. From Fig. 2 and Fig. 3, we can get the same conclusion that FBP achieves the best reconstruction accuracy and robustness among FBP, ASMP and ATBOMP.

Under reasonable conditions, FoBa [15] pointed out that, through a novel combination of these two greedy ideas, an adaptive forward-back greedy algorithmcan effectively solve thesparselearningproblem. Based on the FoBa idea, this manuscript is to investigate the differences among these algorithms and furtherly to propose a new algorithm to improve the performance of adaptive forward-back greedy algorithm.

\section{Proposed Algorithm DTMP}

The main contribution of DTMP is how to decide two proper thresholds for forward and backward processing.

\subsection{The Structure of DTMP}

The DTMP algorithm is initialized with a trivial signal approximation, which means that the initial 
residualequals the unknown target signal. During each iteration, DTMP performs five major steps as follows.

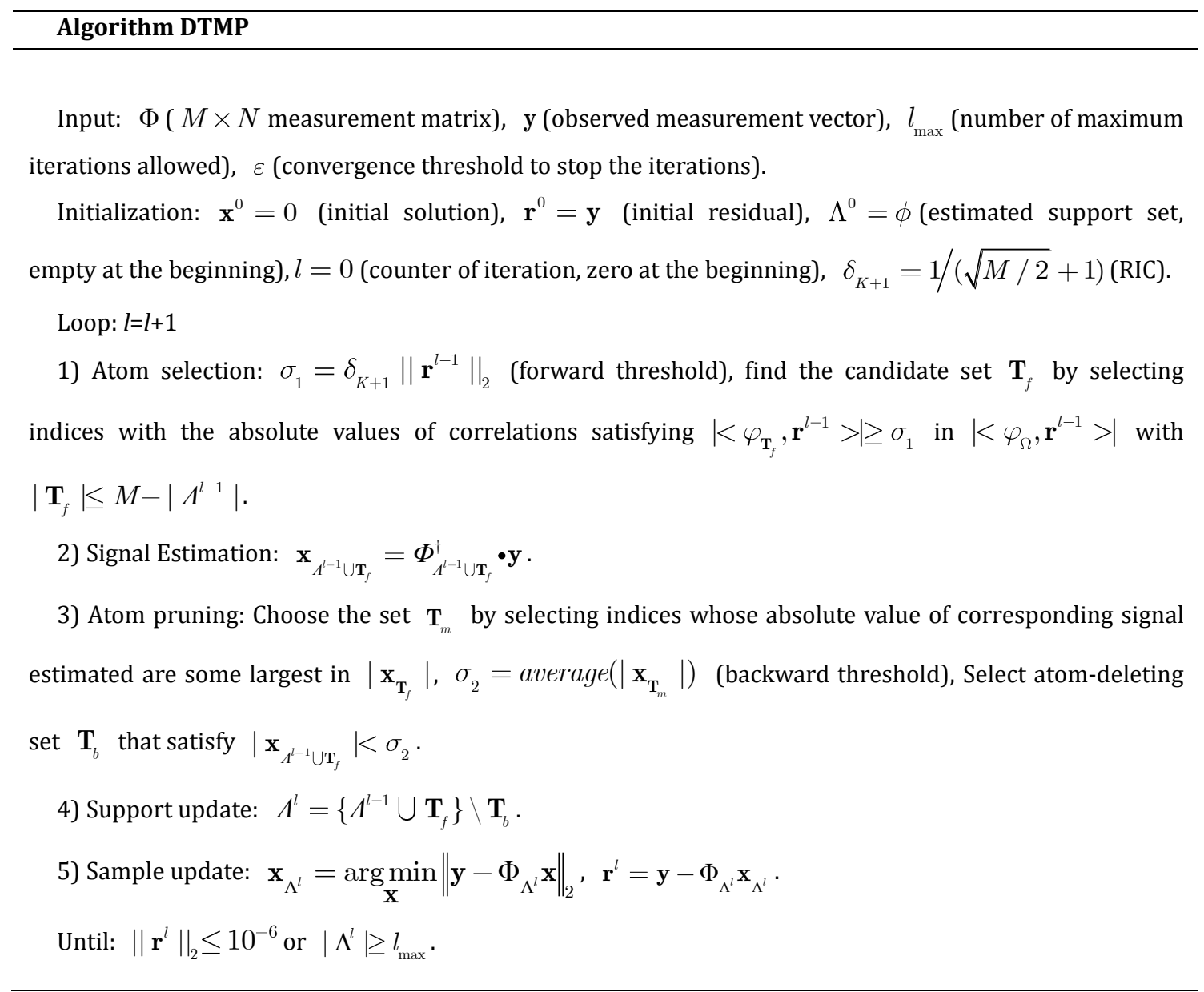

\subsection{Thresholds of DTMP}

Suppose $\Phi \in R^{M \times N}$ satisfies the Restricted Isometry Property with parameter $(K, \delta)$ when $K \leq M$, $0 \leq \delta \leq 1$, any $K$-sparse signal can satisfy [1]-[4] $\left(1-\delta_{K}\right)\|\mathbf{x}\|_{2}^{2} \leq\|\Phi \mathbf{x}\|_{2}^{2} \leq\left(1+\delta_{K}\right)\|\mathbf{x}\|_{2}^{2}$.

Lemma 1: For any integers, $\delta_{m} \leq \delta_{n}$.

Lemma 2: Suppose $\operatorname{supp}(\mathbf{x}), \operatorname{supp}(\mathbf{y}) \subseteq\{1,2, \cdots N\},|\operatorname{supp}(\mathbf{x})| \leq m,|\operatorname{supp}(\mathbf{y})| \leq n, \operatorname{supp}(\mathbf{x}) \cap \operatorname{supp}(\mathbf{y})=\phi$, then $K \Phi \mathbf{x}, \Phi \mathbf{y}>\mid \leq \delta_{m+n}\|\mathbf{x}\|_{2}\|\mathbf{y}\|_{2}$

When $l=1$, suppose $\Phi \in R^{M \times N}$ satisfies the RIP with parameter $(K, \delta)$, from Lemma 2 one has

$$
\forall i \notin \operatorname{supp}(\mathbf{x}) \quad\left|\mathbf{h}^{1}(i)\right|=<\varphi_{i}, r^{0}>=<\varphi_{i}, \mathbf{y}>=\left|<\mathrm{Fe}_{i}, \mathrm{Fx}>\right| \leq \delta_{K+1}\|\mathbf{x}\|_{2}
$$

Similarly under the condition $l>1$, one has

$$
\forall i \notin \operatorname{supp}(\mathbf{x}), \begin{aligned}
& \left|\mathbf{h}^{l}(i)\right|=\left|<\varphi_{i}, r^{l-1}>\right|=\left|<\mathrm{Fe}_{i}, \mathbf{y}-\mathrm{F}_{\mathrm{L}^{L}} \mathbf{x}_{\mathrm{L}^{l}}>\right|=\left|<\mathrm{F} \mathbf{e}_{i}, \mathrm{Fx}-\mathrm{F}_{\mathrm{L}^{L^{\prime}}} \mathbf{x}_{\mathrm{L}^{l}}>\right| \\
& =\left|<\mathrm{Fe}_{i}, \mathrm{Fx}-\mathrm{Fx}_{\mathrm{L}^{l}}>\right|=\left|<\mathrm{F} \mathbf{e}_{i}, \mathrm{~F}\left(\mathbf{x}-\mathbf{x}^{l-1}\right)>\right| \leq \delta_{K+1}\left\|\mathbf{x}-\mathbf{x}^{l-1}\right\|_{2}
\end{aligned}
$$


Without consideration of noise, OMP can guarantee the exact support recovery of $K$-sparse signal if RIP constant satisfies $\delta_{K+1}<1 /(\sqrt{K}+1) \quad[16]$. Generally, $1 /(\sqrt{K}+1)>1 /(\sqrt{M / 2}+1)$, if set $\delta_{K+1}<1 /(\sqrt{M / 2}+1)$, the $\delta_{K+1}<1 /(\sqrt{K}+1)$ is satisfied. Therefore, under the sparsity level of target signal is unknown, we can estimate $\delta_{K+1}$ with $1 /(\sqrt{M / 2}+1) 1 /(\sqrt{M / 2}+1)$. Meanwhile, the observation matrix is normalized. So one has $\left\|\mathbf{r}^{0}\right\|_{2}=\|\Phi \mathbf{x}\|_{2}=\|\mathbf{y}\|_{2} \approx\|\mathbf{x}\|_{2}$. Furtherly, $\|\mathbf{x}\|_{2},\left\|\mathbf{x}-\mathbf{x}^{l-1}\right\|_{2}$ in equation (2), (3) can be replaced by $\left\|\mathbf{r}^{0}\right\|_{2},\left\|\mathbf{r}^{l}\right\|_{2}$ respectively. At last, we obtain the parameter $\sigma_{1}=\delta_{K+1}\left\|\mathbf{r}^{l-1}\right\|_{2}$. $\left\|\mathbf{r}^{l}\right\|_{2}=\left\|\mathbf{y}-\Phi \mathbf{x}^{l-1}\right\|_{2}=\left\|\Phi\left(\mathbf{x}-\mathbf{x}^{l-1}\right)\right\|_{2} \approx\left\|\mathbf{x}-\mathbf{x}^{l-1}\right\|_{2}$

Since the target signal includes only $K$ nonzero entries, we assume that residual after each iteration be a compressible signal whose main information is contained in a small amount of elements. Thanks to the principal of energy concentration, the threshold $\sigma_{2}$ is set to the average absolute amplitude of these several largest elements in the newly reconstructed elements. If the estimated elements indexed by the whole support are smaller than the threshold decided by $\sigma_{2}$, it is highly probable that the corresponding atoms are wrong, which should be deleted to shrink the support.

\section{Experiment}

Based on the MATLAB platform, we test both 1D and 2D signals. In 1D experiment, three different signals, namely, Gaussian random sparse signal, '0-1'sparse signal and uniform signal are used. For the 2D simulation, we use the standard Lena image with size $256 \times 256$. The entries of observation matrix $\Phi$ are drawn from the standard Gaussian distribution.

\subsection{D Signal Reconstruction}

The signal size is fixed to $N=256$, the observation number is fixed to $M=128$ and the degree of sparse is $K \leq M / 2$. The termination thresholds for DTMP and FBP are $\varepsilon=10^{-6}$. Meanwhile the maximum iteration is $l_{\max }=M / 2$. In DTMP, the forward constant is $\delta_{K+1}=1 /(\sqrt{M / 2}+1)=1 / 9 \approx 0.1$ and the threshold $\sigma_{2}$ is set to the average amplitude of $30 \%$ largest elements in amplitude of newly reconstructed elements. The cvx box is applied to run BP algorithm. In FBP, the forward step length $\alpha$ is fixed with $0.3 \mathrm{M}, 0.2 \mathrm{M}$ and the backward step length is $\alpha-1$ respectively. The average run time, exact reconstruction rate and ANMSE curves, which are defined as that in Fig. 1-Fig. 3, are given in Fig. 4-Fig. 6.

Fig. 4 shows the Gaussian signalreconstruction resultsobtained by FBP, SP, BP and DTMP. The second curve in Fig. 4 presents the relationship between the exact reconstruction rate and sparsity. When the sparsity $K$ is relatively small, all algorithms can guarantee $100 \%$ reconstruction. With the increase of sparsity level, some algorithms begin to fail at certain point, from which we can evaluate the stability of an algorithm.It is obvious that BP and SP begin to fail around $K=40$. No matter FBP chooses its forward step length as $\alpha=0.3 \mathrm{M}$ or $0.2 \mathrm{M}$ and back step length as $\beta=\alpha-1$, it begins to fail when $K \geq 50$. For the new DTMP, only when the sparsity $K$ approaches to 60 , its exact reconstruction rate decreases a bit. The third curve in Fig. 1 shows the ANMSE, which can indicate the reconstructionaccuracy of algorithms. It is obvious that the ANMSE of DTMP is the lowestamongall presented algorithms during the whole sparsity set, which is unbelievably low. Finally, from the first curve, namely, average run time, we can measure the reconstruction complexity of each algorithm. DTMP and SP costs similarly short time in the reconstruction process, far shorter than FBP and BP (more than $200 \mathrm{~ms}$, not present in pictures).

Fig. 5 shows the uniformsparse signal reconstruction results. Similarly, DTMP is the best performer that it 
present the best exact reconstruction rate and ANMSE, meanwhile, its time cost is comparable to SP, especially when the sparsity satisfies $100 \%$ successful reconstruction.

In Fig. 6, the reconstruction performance of DTMP under 0-1 sparse signal condition is measured. In exact reconstruction rate curve, as the sparsity $K$ gradually increases from 0 to 37 , SP and FBP $(0.2 \mathrm{M}$, $0.2 \mathrm{M}-1)$ begin to fail when $K$ reaches 28 . FBP $(0.3 \mathrm{M}, 0.3 \mathrm{M}-1)$ fails later at $K=30$ while DTMP keeps $100 \%$ successful reconstruction rate till $K=35$. As for ANMSE, DTMP keeps at the lowest condition, close to BP. In addition, the reconstruction time of DTMP remains quietly short, just as that in Fig. 4 and Fig. 5.
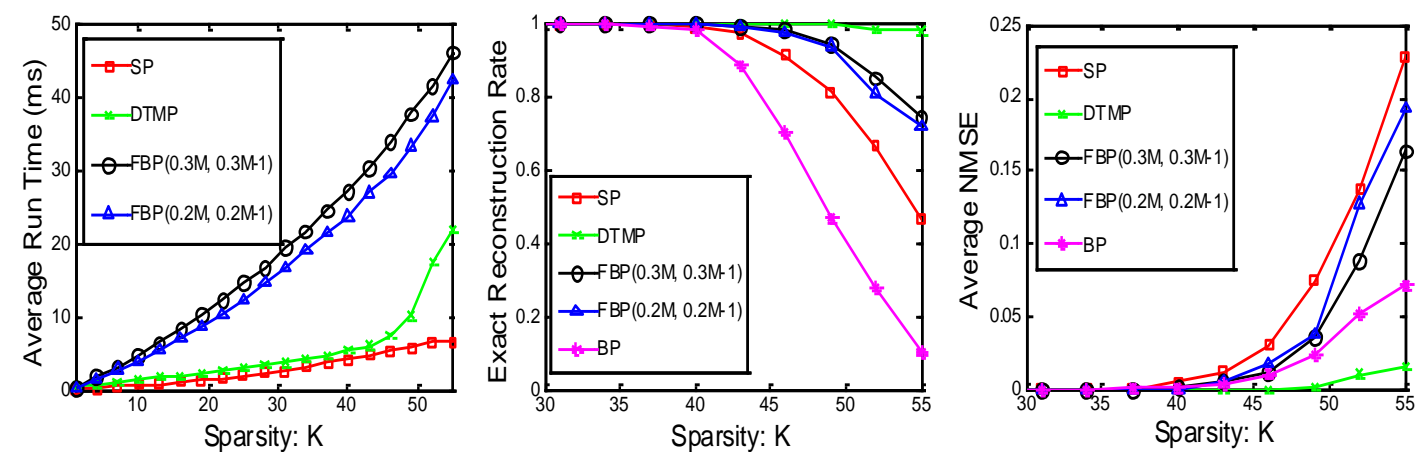

Fig. 5. Reconstruction results over sparsity for the uniform sparse signal.
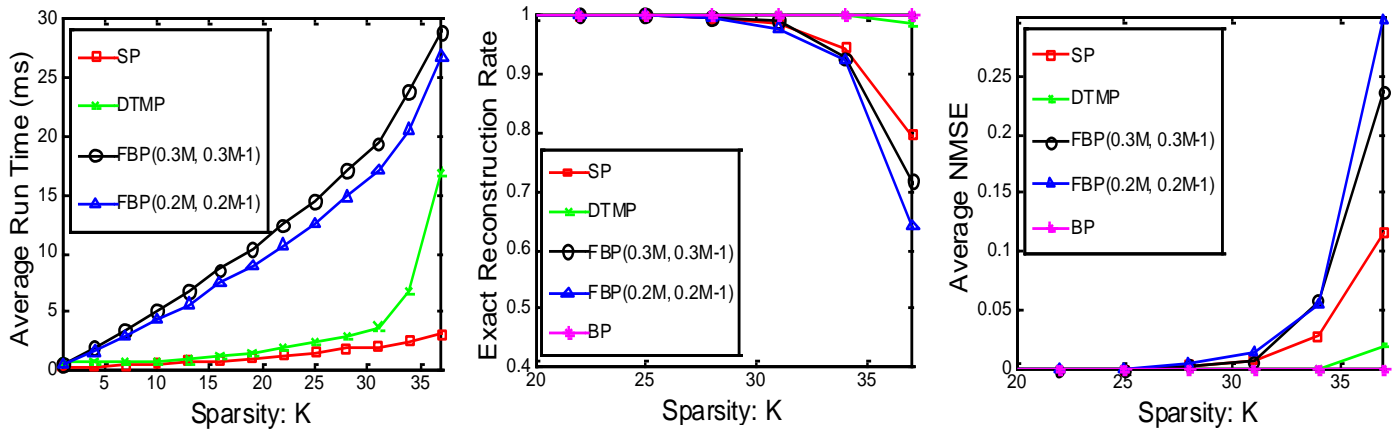

Fig. 6. Reconstruction results over sparsity for the '0-1' sparse signal.

\subsection{D Simulation}

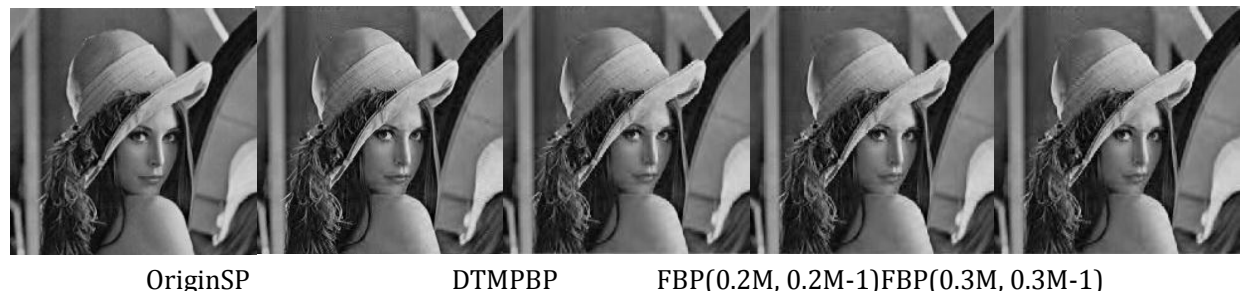

Fig. 7. Reconstruction results of Lena.

Table 1. The PSNR(dB) and Reconstruction TIME(s) of Reconstruction

\begin{tabular}{cccccc}
\hline \hline & BP & DTMP & SP & FBP(0.3M, 0.3M-1) & FBP $(0.2 \mathrm{M}, .2 \mathrm{M}-1)$ \\
\hline PSNR(dB) & 33.66 & 34.03 & 32.79 & 33.71 & 33.25 \\
TIME(s) & 58.56 & 2.11 & 1.20 & 7.72 & 6.90 \\
\hline
\end{tabular}

Lena $(256 \times 256)$ is used to measure the performance of the algorithms for image signal reconstruction. As is known, the CS theory is based on the condition that the signal has a sparse representation in a known 
transform domain $\Psi$ (e.g, the DCT and the wavelet transformation). However, the matrix of wavelet coefficients of image signal is not sparse strictly in sym 6 wavelet basis. K maximum wavelet coefficients of each column is retained with the rest set to zeros so that the sparsity of image is $k$. We set $k=42$ in the following test. Peak signal to noise ratio (PSNR) is used to measure the accuracy of reconstruction result. Fig. 7 and Table 1 represent the reconstruction results of 'lena'. We can find that DTMP gets the most accurate reconstruction as well as comparatively low compute complexity. In conclusion, DTMP performs betterinthe image signal reconstruction processthan other algorithms.

\section{Conclusion}

This paper presents a new adaptive forward-back greedy algorithm calledDual Threshold Matching Pursuit. Simulation results show that for a Gaussian signal, DTMP reconstruction accuracy is better than all algorithmsincluding FBP and in the whole sparse interval, reconstruction error is maintained at a quite low level; For the ' $0-1$ ' signal, reconstruction results of DTMP significantly isbetter than any other greedy algorithms, close to BP. For the image signal, DTMP not only reconstruction accuracy is higher than all the other algorithms, buttime consumption is the least in a certain condition.

\section{Acknowledgements}

This work was supportedby the National Natural Science Foundation of China (61171077).

\section{References}

[1] Donoho, D. L. (2006). Compressed sensing. IEEE Transactions on Information Theory, 52, 1289-1306.

[2] Baraniuk, R. G., et al. (2008). Compressive sampling. IEEE Signal Processing Magazine, 25.

[3] Candes, E. J., \& Wakin, M, B. (2008). An introduction to compressive sampling. Signal Processing Magazine, 44, 21-30.

[4] Duarte, M. F., \& Eldar, Y. C. (2011). Structured compressed sensing: From theory to applications. IEEE Transactions on Signal Processing, 59, 4053-4085.

[5] Kim, S. J., et al. (2007). An interior-point method for large-scalel (1)-regularized least squares. IEEE Journal of Selected Topics in Signal Processing.

[6] Candes, E. J. (2008). The restricted isometry property and its implications for compressed sensing. Comptes Rendus Mathematique, 346, 589-592.

[7] Tropp, J. A., \& Gilbert, A. C. (2007). Signal recovery from random measurements via orthogonal matching pursuit. IEEE Transactions on Information Theory, 53, 4655-4666.

[8] Dai, W., \& Milenkovic, O. (2009). Subspace pursuit for compressive sensing signal reconstruction. IEEE Transactions on Information Theory, 55, 2230-2249.

[9] Needell, D., \& Tropp, J. A. (2009). CoSaMP: Iterative signal recovery from incomplete and inaccurate samples. Applied and Computational Harmonic Analysis, 26, 301-321.

[10] Karahanoglu, N. B., \& Erdogan, H. (2013). Compressed sensing signal recovery via forward-backward pursuit. Digital Signal Processing, 23, 1539-1548.

[11] Honglin, W., \& Shu, W. (2012). Adaptive sparsity matching pursuit algorithm for sparse reconstruction. Signal Processing Letters, 19, 471-474.

[12] Zhao, H., \& Zhang, G. (2013). Adaptive threshold backtracking matching pursuit for compressive sensing. Proceedings of IET International Radar Conference (pp. 1-4).

[13] Do, T. T., et al. (2008). Sparsity adaptive matching pursuit algorithm for practical compressed sensing. Proceedings of 42nd Asilomar Conference on Signals, Systems and Computers (pp. 581-587).

[14] Honglin, H., \& Makur, A. (2011). Backtracking-based matching pursuit method for sparse signal 
reconstruction. Signal Processing Letters, 18, 391-394.

[15] Tong, Z. (2011). Adaptive forward-backward greedy algorithm for learning sparse representations. IEEE Transactions on Information Theory, 57, 4689-4708.

[16] Chang, L. H., \& Wu, J. Y. (2014). An improved rip-based performance guarantee for sparse signal recovery via orthogonal matching pursuit. IEEE Transactions on Information Theory, 60, 5702-5715.

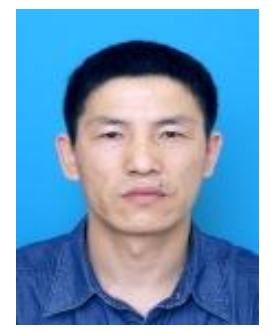

Z. G. Xie received his $\mathrm{PhD}$ degree in communications and information system from Beijing University of Post and Telecommunications, in 2007. He is now a professor with Nantong University. His research interests include communications signal processing, wireless communication, image and video compression.

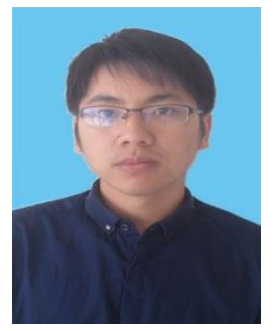

H. W. Huang is currently pursuing the master degree in information and communications engineering at Nantong University. His research interests include communications signal processing, image and video compression.

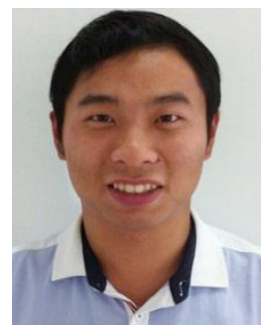

X. Cai is currently pursuing the master degree in information and communications engineering at Nantong University. His research interests include communications signal processing, image and video. 